MUZIKOLOS̃KI ZBORNIK - MUSICOLOGICAL ANNUAL XVI, LJUBLJANA 1980

UDK 78.01:659.3

\title{
MUSIK ALS AUSDRUCKS- UND MITTEILUNGSSYSTEM
}

\author{
Josef B e k (Praha)
}

Die steinalte Aporie der Musikästhetik, die das Wesen der Musik einerseits in der außerhalb des Menschen stehenden objektiven und andererseits in der spezifisch menschlichen Gesetzmäßigkeit sucht, hatte in onthischer Ebene bis etwa ins 19. Jahrhundert hinein immerhin etwas gemeinsam: sie faßte die Musik als Trägerin einer Bedeutung, als Kommunikationsmittel auf. Erst die Krise des bürgerlichen Bewußtseins brachte eine die Semantik der Musik negierende und deren Sinn in sie selbst verschließende Konzeption hervor.

Die Musikästhetik, anknüpfend an fortschrittliche Traditionen materialistischer Philosophie, Ästhetik und Kunsttheorie, etwickelt jene Auffassung, die bereits im klassischen Altertum der Kunst eine gesellschaftlich aktivierende Rolle beigemessen und sie als unvertretbares Instrument des Menschen zur Aneignung der Welt und deren Umgestaltung verstanden hatte. Im Interesse der vollständigsten Ausnutzung der Kunst im Kampfe um den gesellschaftlichen Fortschritt fiel der marxistischen Ästhetik die Aufgabe zu, die Gesetzmäßigkeiten der Kunst, deren Wesen und Existenzformen, die Grundmechanismen ihres gesellschaftlichen Funktionierens, kurz die spezifische Rolle der Kunst in der Gesellschaft $\mathrm{zu}$ untersuchen und wahrheitsgetreu $\mathrm{zu}$ beleuchten. Die von der also orientierten Ästhetik untersuchte Aufgabe kann nie endgültig gelöst werden, was keine Konstatierung eines allgemein gültigen Prinzips ist, sondern eine aus der veränderlichen Natur des Untersuchungsgegenstandes selbst (dh. der Kunst) und der verschiedensten Anschauugns- bzw. Wahrnehmungsarten der Kunst vonseiten des Konsumenten folgert. So wie der Künstler auf die sich stets verändernde Wirklichkeit reagiert und einen adäquaten Kunstausdruck dafür sucht, so ist auch das Herangehen des Wahrnehmers ans Kunstwerk durch Änderungen der gesamten Kultur, Denk- und Gefühlsweise der Zeit beeinflußt. Lapidär gesagt ist das Herangehen an die Kunst inmitten der wissenschaftlich-technischen Revolution ein anderes als zu Marxens Zeiten. 
Alle diese Tatsachen zwingen die marxistische Ästhetik und Kunstheorie, die des Namens wert sein will, immer neue Zugangswege an die Lösung ihrer Aufgabe $\mathrm{zu}$ suchen. Das gnoseologische und ontologische Fundament sichert der marxistischen Ästhetik und Kunsttheorie genug Mittel, jede fruchtbare Anregung zu ihrem Vorteil $\mathrm{zu}$ wenden, gleichviel ob jene aus einer materialistisch oder idealistisch orientierten Auffassung herkommt. Wenn der Marxist einen festen Standpunkt einnimmt, erübrigen sich Befürchtungen um »Reinheit« der eigenen Konzeption und es besteht auch kein Grund, diesen oder jenen Forscher des Modewahns zu bezichtigen. Es ist im Gegenteil eine notwendige Aufgabe, die der marxistischen Ästhetik fortwährenden Kontakt mit lebendiger Kunstproblematik sichert.

Dies gilt ebenfalls von der Semiotik, die aus linguistischen Kreisen unter die Literaturwissenschaftler, allgemeinen Kunsttheoretiker und bis $\mathrm{zu}$ den Musikologen vorgedrungen ist. Ihre Verwandschaft mit der Kybernetik bzw. Informationstheorie (die allerdings erst später entwickelt worden sind) reiht die Semiotik eher den mathematischlogischen als den Gesellschafts-wissenschaften an und hat demnach in ihrer ursprünglichen Gestalt überideologischen Charakter. Ideologische Intaktheit bringt Vorteile wie auch Nachteile. Sie kann im Grunde jedermann ihre Dienste anbieten, doch birgt gleichzeitig die Gefahr einer klassenlosen Sicht in sich, dort, wo die "Klassenlosigkeit« eine falsche Interpretation der Wirklichkeit darstellt. Dies z. B. ist das Problem, an dem im Laufe der dreißiger Jahre die tschechischen Strukturalisten scheiteten, wiewohl sie sich politisch auf das linke Flügel der sozialistischen Avantgarde stellten.

Mit anderen Worten, Anregungen der Semiotik können für die marxistischen Ästhetiker, Kunsttheoretiker, Musikwissenschaftler usw. nur dann fruchtbar werden, wenn man keinen Augenblick die Grundsätze des dialektischen und historischen Materialismus aus der Sicht verliert, die heutzutage bereits zu einer verhältnismäßig abgerundeten kunstwissenschaftlichen Methodologie ausgearbeitet sind. Gleichzeitig ist eine prinzipielle Übereinstimmung zwischen marxistischem und semiotischem Herangehen in musikologischer Ebene wahrzunehmen: in der Auffasung der Musik als eines spezifischen Kommunikationssystems, als Trägerin eines Inhaltes. Wiewohl diese Auffassung von der Musik so alt wie die Musik selbst ist, tauchen immer wieder Fragen auf, die nicht ganz befriedigende Antwort finden. Es geht nun darum festzustellen, ob die Semiotik zur Lösung solcher Fragen positiv beitragen kann.

Die marxistisch orientierte Musikologie hat sich durch etliche ihrer Konzeptionen den Boden zur Aufnahme semiotischer Inspiration vorbereitet. $\mathrm{Al}$ s ein bedeutender Versuch dieser Art kann die Intonationstheorie von B. W. Asafjew angesehen werden. Einen Schlüsseltermin für die Auffindung von Korrelaten mit semiotischer Termino- 
logie stellt die »Intonation« als musikalisch-semiotische Einheit dar, die durch die tschechischen Forscher Jaroslav Jiránek und Jaroslav Volek definiert worden ist. Ihnen zufolge gleicht die Intonation einer Menge kleinster konkret-musikalisch bestimmter Kontexte, welche für ein historisch-konkret bestimmtes gesellschaftliches Subjekt relativ gleiche Bedeutung und gleichen Ausdruckswert verweisend zu gleicher gesellschaftlicher und Lebenssituation und zu gleichem Umkreis außer musikalischer Wirklichkeit) besitzt, charakterisiert durch bestimmte konkrete Wahrscheinlichkeitsstruktur von Beziehungen innerhalb aller und unter allen Komponenten der musikalischen Äußerung: der melodischen, rhytmischen, harmonischen, timbrehaften, dynamischen, des Tempos und des Vortrags. ${ }^{1}$ Die alle einzelnen Gedanken Asafjews zusammenfassende und zu Ende denkende Definition zeugt eindeutig dafür, daß der Autor der Intonationstheorie die Musik als Ausdrucks - und Mitteilungssystem, dienend der menschlichen Erkenntnis und Umgestaltung der Wirklichkeit verstand. Der marxistische Standpunkt, von der Widerspiegelungstheorie ausgehend, ist hier auf Annahme semiotischer Impulse »gestimmt«.

Es ist jedoch nicht nur Asafjew, der bei der Klärung der musikalischen Bedeutung und des Sinnes der Musik an solche Erfassung der Probleme nahekam, die auch für die Semiotik bezeichnend ist. Eine ähnliche Forschungsrichtung nehmen auch weitere sowjetische Musikologen ein, so L. A. Mazel und V. A. Cukkermann (Analiz muzykalnych proizvedenij. Elementy muzyki i melodika analiza malych form, 1967), die polnische Forscherin Z. Lissa (Podstawy marksistowskej estetyki muzycznej, I, II, 1954; Fragen der Musikästhetik, 1954), der Magyare J. Ujfalussy (A valósáy zenei képe, 1962), der deutsche marxistische Musikästhetiker H. Goldschmidt (Um die Sache der Musik, 1970) und allerdings auch tschechische Forscher mit Antonín Sychra an der Spitze, denen Mukařovský strukturalistische Schule Vermittlerin war, selbstverständlich in einer streng kritischen Umwertung. Es ist bekannt, daß einige der genannten Forscher in verschiedenen Ländern, durch das Bestreben um Ausarbeitung der Widerspiegelungstheorie in der Musik geleitet, schon vor 15-20 Jahren die allgemeine Semiotik aufgegriffen haben, ausgehend namentlich von Arbeiten des amerikanischen Logikers und Semiotikers Ch. S. Peirce. Gegenwärtig kann festgestellt werden, daß eine selbständige Disziplin aus der $\mathrm{Mu}$ sikologie als wissenschaftlichen Fachgebietes sich auszusondern beginnt - die musikalische Semiotik, und wenn man die Entwicklung der Musikwissenschaft global betrachtet, so kann man hinzufügen, daß die soeben genannten Musikologen einen beträchtlichen Anteil an der Entstehung der neuen Disziplin nehmen.

1 Vgl. J. Jiránek, Asafjevova teorie intonace, její geneze a význam (Asafjews Intonationstheorie, deren Genesis und Bedeutung), Prag 1967, S. 235 . 
Eine ausgiebige Stütze bietet den tschechoslowakischen Musikforschern die fortgeschrittene Ausarbeitung semiotischer Herangehen in der allgemeinen Kunsttheorie, besonders in dem Prager interdisziplinären Team für Erförschung der Ausdrucks und Mitteilungssysteme der Kunst, der seit dem Jahre 1971 arbeitet. ${ }^{2}$ Zur Ausgangsthese des genannten Teams wurde Marxens Gedanke von der Kunst als einer besonderen Form von Weltaneignung durch den Menschen. Der Aneignungsprozeß umfaßt sowohl Erkenntnismomente als auch Momente gegenständlichen Schaffens und subjektiver Bereicherung des Menschen. Die Kunst erfüllt die Aneignungsfunktion einer besonderen Art Mitteilung (Kommunikation); sie spricht nicht nur eine Einzelfunktion des Menschen sondern den ganzen Menschen an, den Gesamtkomplex seiner psychischen Kräfte (Verstand, Gefühl und Wille), die sie reorganisiert und zu neuer Harmonie führt. Für die Kunst gilt im allgemeinen, daß sie im Weltaneigungsprozeß vor allem drei Hauptfunktionen erfüllt: die erkenntnishafte (die Kunst erwirbt und gestalten auf besondere Art Erkenntnisse über den Menschen und die Welt), harmonisierende und bereichernde (die Kunst vereinigt die psychischen Kräfte) und verständigende (die Kunst stellt ein besonderes Mittel der Nachrichtenübermittlung, menschlichen Kommunisation dar, indem sie die Menschen zur praktischen Tätigkeit einigt).

Für ein jedes Kunstwerk gilt, daß es eine Widerspiegelung objektiver Wirklichkeit darstellt. Daraus folgt, daß das Kunstwerk imstande ist, die Gesamtheit menschlicher Psychik auf etwas anderes zu richten als das Werk selbst, ihm eignet also Zeichencharakter. Die Kunst interessiert sich jedoch nicht an der Wirklichkeit in ihrer relativen Autonomie, als etwas nur für passive Anschauung des Menschen geeignetes, sondern in der Beziehung zur Welt des Meschen, in Einheit mit der menschlich gesellschaftlichen Praxis, gerichtet auf Umgestaltung der Wirklichkeit nach menschlichen Vorstellungen.

Gegenstand der künstlerischen Widerspiegelung ist also die vermenschlichte Welt, in der das gesellschaftliche Sein sich bereits abgedruckt hat. Dabei ist zu unterscheiden zwischen dem Gegenstand künstlerischer Widerspiegelung und dem Sujet des Kunstwerkes. Zum Sujet kann ein bestimmtes Sein, ein Wirklichkeitsausschnitt werden, während den Gegenstand künstlerischer Widerspiegelung die Realität menschlichen Seins in ihrer Totalität ausmacht. Damit hängt auch die spezifische Stellung des schaffenden Subjekts zusammen, welches die Realität in der Gesamtheit ihrer individuellen und sozielen Charakteristiken widerspiegelt; das schaffende Subjekt ist nun kein außerhalb des sozialen Kontextes stehendes Ausmahmeindividuum, sondern ein

2 Der Interdisziplinäre Team wird von Sáva Šabouk geleitet, der auch Autor einiger Arbeiten ist; am bedeutendsten davon »Umění, systém, odraz" (Kunst - System - Widerspiegelung), wurde ins Russische übersetzt (Moskau 1976). 
konkretes Mitglied einer Gesellschaft, welches durch seine Lebenserfahrung einen Teil kollektiver Erfahrungen der Menschheit, des historischen Prozeßes wechselseitigen Durchdringens von Subjekt und Objekt sich angeeignet hat. Durch aktive Teilnahme an konkreter Lebenspraxis seiner Zeit ist der Künstler fähig, ein bestimmtes Geschenen in der Wirklichkeit $\mathrm{zu}$ widerspiegeln und die im Kunstwerk zu lösenden Probleme aufzudecken. Indem er an diesem als menschliche Praxis bezeichneten Geschehen durch sein ganzes Wesen (zum Unterschied etwa vom Wissenschaftler) mit allen seinen psychischen Kräften teilnimmt, kann die künstlerische Widerspiegelung vermenschlichter Wirklichkeit nur als ein Ergebnis rationaler Erkenntnis erklärt werden.

Die Besonderheit der künstlerischen gegenüber der wissenschaftlichen Erkenntnis liegt vor allem darin, daß sie unmittelbarer auf das Geschehen menschlicher Praxis gezielt ist und daß sie eine andere Struktur des zu widerspiegelnden Gegenstandes, gegründet auf Interferenz von Subjekt und Objekt, aufweist. Die künstlerische Erkenntnis eröffnet komplexe Einsicht in die Welt menschlicher Existenz und legt ein dynamisches Bild konkreten Geschehens menschlicher Praxis als dialektischer Einigung des Menschen mit der Welt vor. Sie trifft den Menschen auf eine mehr komplexe und zugleich individuelle Art als die Wissenschaft, indem sie soweit individualisiert ist, daß der jeweilige Empfänger sie als seine eigene Erkenntnis und die vom Kunstwerk gelösten Probleme als seine eigenen Probleme hinnimmt. Dies ist einer der wessentlichen Züge künstlerischer Erkenntnis; doch darüber hinaus, diese Erkenntnis ereignet sich im Prozeß des Schaffens und im Wahrnehmungsprozeß eines Kunstwerkes. Die vom schaffenden Künstler während der Herstellung des Kunstwerkes erreichte Erkenntnis wird in die Erkenntnis des Wahrnehmers im Laufe der Aperzeption des Werkes strukturell transformiert. Der Wahrnehmende wird somit zum Interpreten, überdies auch zum aktiven Mitgestalter des Werkes. Im Falle Musik tritt in diesem Prozeß ein weiterer Faktor ein - der interpretierende Künstler, im Falle des Theaters, Films udg. ist dieser Prozeß noch komplizierter.

Einen wichtigen Ausgangspunkt des Prager interdisciplinären Teams bildet die Auffassung von der Kunst als System. Die Kunst in ihrer Ganzheit nicht als bloße Summe von Kunstwerken bzw. von verschiedenen mit der Kunst verknüpften Aktivitäten begriffen werden; wir könnten solcherart nicht zum Wesen und Sinn der Kunst in der Gesellschaft vordringen. Für richtiges Verstehen der Kunst ist es notwendig dynamische Beziehungen zwischen allen Elementen zu untersuchen, die in ihrer Gesamtheit einen innerlich verknüpften Organismus bildet. Die Grundelemente, genauer gesagt Mikrostrukturen sind: der Künstler - das Werk - die künstlerische Tradition - der Wahrnehmer. Der dynamische Komplex aller Mikrostrukturen, deren 
wechselseitige Verknüpftheit und Gegenaktivität bildet ein gesetzmäßig geregeltes System der Kunst, welches dadurch charakteristisch ist, daß es sich fortwährend ereignet, entwickelt und reorganisiert und $\mathrm{da} \beta$ es fortwährend in das breitere System menschlichen Seins und objektiver Wirklichkeit hinein sich öffnet.

Wenn man unter den Grundelementen des Kunstsystems die künstlerische Tradition hervorhebt, ist es deshalb, weil die Kunst ähnlich wie die Wirklichkeit im Prozeß der Weltaneigung auch von ihren eigenen angesammelten Erkenntnissen und Erfahrungen, wie auch der künstlerischen Tradition Nutzen zieht, welche fest in das Gesamtsystem der Kunst eingeschaltet ist. Die Schaffenden und deren Werke leben nicht verschlossen gegenüber Einflüssen anderer KunstWerke und Kulturtraditionen. Dies gilt mit obensolchem Recht von den ausübenden Künstlern oder Hörern, die den Inhalt eines neuen Kunstwerkes intepretieren und konkretisieren, dies nicht nur mittels persönlicher Lebenserfahrungen, sondern auch ihrer eigenen vorangegangenen Begegnungen mit anderen Kunstwerken der Gegenwart und Vergangenheit. Einzelne Werke näheren einander aufgrund innerer Verwandtheit, verdeutlichen und vertiefen einander inhaltlich (der Prozeß verläuft nicht von der Vergangenheit in die Gegenwart, sondern auch umgekehrt) und somit höhere inhaltliche Synthesen zeitigen. Festes Bewußtsein dieses wechselseitigen Geschehens zwischen Werken der Gegenwart und Vergangenheit stellt die Grundlage der Tradition her, die Kontinuität und eine gewisse Logik künstlerischer Entwicklung garantierend. Je tiefer und inhaltsreicher das Bewußtsein der Tradition, desto kräftiger kann jedes einzelne Werk wirken, desto bedeutender kann es eine aktivierende und harmonisierende Rolle im Aneignungsprozeß der menschlichen Wirklichkeit spielen. ${ }^{3}$

Die Traghaftigkeit eines Systemauffassung der Musik bewährt sich u. a. sehr viel in der musikalisch-historiographischen Forschung. Die Dynamik eines Systems macht auf fortwährende gesellschaftlichhistorisch bedingte Inhaltsveränderlichkeit aufmerksam, auf deren kontinuierliche innere Differenziertheit, typologische Schichtung und Entwicklungspolarisation usw.

3 In der Musikgeschichte bietet der Neoklassizismus ein hervorstechendes Beispiel für das Prinzip höherer Inhaltssynthesen und der Einschaltung der Kunsttradition in das System der Musik. Gegen die ungerechte Kritik T. W. Adornos in der bekannten »Philosophie der neuen Musik« (1. Aufl., Tübingen 1949) kann eben auf diesen Grundlagen argumentiert werden. Dies ermöglicht, den historischen Beitrag des Neoklassizismus zu begreifen, der zum Unterschied von anderen avantgardistischen Bewegungen der 20. Jahre die Devise einer aktiven Rückkehr zu etwas prägte, was in dem musikalschen Halbbewußein als sinnvoller, semantisch und inhaltlich konkretisierter Verständigungscode verankert war. Der Neoklassizismus präsentiert sich in diesem Licht als bewußt gezielte Bewegung für schaffende Ausnutzung historisch aufgeschichteter Werte, für deren Einschaltung in die Lösung gegenwartiger Probleme menschlicher Existenz. 
Sofern man aller Art gesellschaftliche Aktivitäten des Menschen in vier elementare Formen (umgestaltende, wertende, orientierende und kommunikative Tätigkeit) gliedern kann, dann gilt für die Kunst - wie schon angedeutet wurde - daß alle diese Formen darin organisch zu einem mehrfunktionellen Systemganzen zusammenwachsen. Dies stellt einen bedeutenden spezifischen Zug jeder Kunstart mitsamt der Musik dar, obwohl das Maß der Beteiligung einzelner Formen verschieden und zudem - historisch veränderlich ist. Das Studium wechselseitiger Relationen innerhalb einer Epoche, des Werkes eines einzelnen Komponisten kann zur Klärung einer Reihe historisch relevanter Fragen beitragen. Die Botschaft von Beethovens Werk besitzt zweifellos eine starke noetische Ladung. Durch tiefes Eindringen bis zu den Wurzeln menschlicher Existenz enthüllte der Komponist seinen Zeitgenossen neue Erkenntnis, die das konventionelle Weltbild und die Stellung des Menschen in dieser Welt zerstört hat. Der Illusionsverlust ist immer schwer $\mathrm{zu}$ tragen und neue Ideen werden nicht leicht durchgesetzt. Diese Erfahrung hat auch Beethoven erprobt. Es geht jedoch nicht nur um soziale Funktionen oder gar nur um Beethovens gesellschaftliche Stellung. Das Anwachsen gewisser Aktivisierungsformen innerhalb eines Systems beeinträchtigt den aktiven Anteil anderer Formen, die einen steigen gleichzeitig mit der besonders exponierten Form auf, andere hinwieder treten zurück. Das Bestimmen wechselseitiger Bindungen im System eines Werkes kann nicht minder interessante Erkenntnisse sowohl dem Historiker als auch dem Theoretiker bringen.

In der Gegenrichtung unserer Erwägungen kann wiederum gesagt werden, daß die Gegenwart aller Elementarformen menschlicher Aktivität im System der Kunst verschiedene Austritte in die außerkünstlerische Sphäre ermöglicht und der Kunst innerhalb dieser Suprasysteme (für die Musik in höchster Instanz der gesamten menschlichen Kultur) organische Funktionalität und Sinnhaftigkeit sichert.

Wenn man sich an das von uns gewählte Beispiel hält, so liefert das Sich-Auseinandersetzen mancher Philosophen mit der Gedankenwelt Ludwigs van Beethoven den Beweis dafür.

Die Einschaltung des Werkes auf die Wirklichkeit hin wird durch Eintrittselemente gesichert, die in die Bedeutungsstruktur des Werkes Segmente menschlicher Erfahrung hineintragen, die noch vor der Begegnung mit dem Werke gegenwärtig und im allgemeinen fixiert waren. Sie garantieren eine Harmonie zwischen individueller und Gattungserfahrung und stellen somit eine Voraussetzung für Verständigung zwischen Schöpfer und Wahrnehmer, indem sie in das Werk das hineintragen, was beiden gemeinsam ist. Ein Bestandteil dieser Lebenserfahrungen ist auch die bisherige Erfahrung mit Kunst - die künstlerische Tradition. Auch daher generieren semantische Eintrittsele- 
mente und verweisen durch ihren Eintritt in das Werk auf diese Erfahrung.

Durch Einfügen von semantischen Eintrittselmenten in das Gesamtsystem des semantischen Geschehens eines Werkes entstehen neue Einzelbedeutungen, die nur dem jeweiligen Kunstwerk eigen sind die Semanteme. Für sie ist eine beunruhigende Spannung charakteristisch, welche den Wahrnehmer zur Teilnahme an der Erschaffung neuer Erfahrung, zum Überwinden der entstandenen Distanz, zur Aufdeckung ihrer Wurzeln, die in den Distanzen menschlichen Seins bestehen, auffordert. Das Semantem liefert dabei keine fertigen Erkenntnisse, sondern projektiert den Weg zu ihnen, den der Wahrnehmer selbst durchgehen muß, wie es im Leben auch so ist. An dessen Ende findet sich die projektierte Lösung des gegebenen Problems und eine neue harmonisierung und Aktivisierung der psychischen Kräfte des Wahrnehmers. Das gesamte dynamische System von Semantemen wird somit zur Grundlage der höchsten Schicht inhaltlichen Geschehens des Werkes, dessen ideellen Inhalts.

Aus dieser äußerst kargen Auswahl der allgemeinsten Thesen des Prager interdisziplinären Teams wird deutlich, daß diese Auffassung auch in der Musik erfolgreich anwendbar ist. Insbesondere in der Ebene höherer künstlerischer Allgemeingesetzlichkeiten bietet sich hier eine ganze Reihe Erkentnisse, die auch zur Musik sich beziehen. Nichtsdestoweniger hat jede Kunst ihre eigenen Besonderheiten, von anderen Kunstarten unterschiedlich und bei der künstlerischen Aneignung der Wirklichkeit unvertretbar. Man würde sich der Vulgarisierung schuldig machen, wenn man alle Erkenntnisse der allgemeinen Kunsttheorie mechanisch restlos auf die spezifische Problematik der Musik beziehen wollte. Im Interesse wahrer Erkenntnis der Wirklichkeit ist im Gegenteil immer wieder das Bedürfnis nach gesunden kritischen Mißtrauen $\mathrm{zu}$ betonen, welches jeder Anwendungsoperation beiwohnen muß.

Die Musikästhetik hilft sich schon traditionell bei der Untersuchung der Frage nach Inhaltlichkeit der Musik dadurch, daß sie die Relationen zwischen Musik und Sprache prüft. Es ist dem so auch bei der Erforschung des semantischen Charakters der Musik, wo das bekannte Begriffspaar langue - parole (Sprache - Rede), definiert von de Saussure, ${ }^{4}$ öfters zum Ausgangspunkt wird. Wenn man von dieser Definition Ausgang nimmt, kann man auch in der Musik

4 In der weiteren Behandlung des Themas stütze ich mich auf die neueste Arbeit von J. Jiránek: Tajemství hudebního významu (Geheimnis der musikalischen Bedeutung), Praha 1979, 164 S., die in der tschechoslowakischen Musikologie den ersten Versuch einer systematischen Verwendung der Semiotik für die Musiktheorie darstellt. Gegenwärtig wird die Arbeit auf dem Boden des Instituts für Theorie und Geschichte der Kunst der C̆SAV in Prag diskutiert, wo eine umfangreichere Publikation über die Musiksemiotik vorbereitet wird. 
eine entsprechende Analogie durchaus finden. Statt langue steht hier die gesellschaftlich institutionalisierte und relativ stabilisierte Sphäre musikalischer Bedeutungen, bezeichnet zuweilen als Intonationslexikon der Zeit. Dieses Lexikon umfaßt das allgemeine Moment der Phylogenese, die objektive historische Entwicklung, gegründet auf individuellen Veränderungen. Die Rede (parole) vertritt jede sinnlich konkrete individuelle Musikäußerung (das Werk), worin das Element der Ontogenese als individuelles und relativ abgeschlossenes Ergebnis einer vorangehenden Entwicklung sich manifestiert. Einzelne Musikäußerungen stellen die sinnlich konkrete Sphäre der Musikexistenz dar, Saussures Antinomie langue - parole entspricht somit völlig auch dem Ergreifen grundsätzlicher Musikantinomie.

Etwas anders ist dem jedoch schon in der Frage der Beziehung zwischen objektiven Normen und deren subjektiver Anvendung. Im Falle der Sprache stellt langue das System objektiver Normen dar, welche die richtige Aussprache, den gebührigen Wortvorrat und die richtigen Sprachbindungen bestimmen. In der Sphäre parole kommt es dann zu subjektiver Anwendung dieser Normen, was sich durch bestimmte Intonation der Rede, Wahl bestimmter Wörter und Bindungen, selbstverständlich in gewisser funktionellen Beziehung zum konkreten Kommunikationsbedürfnis, äußert. Fügen wir noch hinzu, daß in der sprachlichen Redeäußerung man von der phonologischen Seite absehen kann, wodurch die Möglichkeit schriftlicher Äußerung sich bietet. Langue - Intonationslexikon der Zeit ist ein System allgemein gültiger und ihrer Bedeutung nach objektivisierter Normen, bzw. Intonationen von Gengre, Gattung, Tektonik und Stil. Es ist offenbar, $\mathrm{da}$ in der Musik keine der genannten Intonationen vor der sinnlichkonkreten Klangform getrennt werden kann. Außerdem gilt auch für sie - ähnlich wie bei anderen Künsten - eine enge weiterer wesentlicher Abweichungen, die das Spezifische künstlerischer Mitteilung begründen. In der musikalischen Äußerung kommt es zu schöpferischer Individualisation einer phylogenetisch vermittelten Struktur von Intonationen mit Rücksicht auf bestimmte inhaltliche Mitteilung. Der Schöpfer eines musikalischen Kunstwerkes führt nie eine bloße Auswahl aus dem Intonationslexikon durch, sondern strebt nach dessen Besonderung, nach Erschaffung einer Spannung, die vom Empfänger im Interpretationsprozeß aktives Herangehen fordert.

Die schon erwähnte musikalische Analogie zwischen langue und parole klingt anders aus, wenn man nach dem Problem des Allgemeinen und Besonderen, Objektiven und Subjektiven fragt. In der sprachlichen Kommunikation ist die individuelle Rede von der allgemeinen langue hergeleitet, die die kollektive Erfahrung der Menschheit zusammenfaßt und maximal objektivisiert. In diesem Sinne hat langue Vorzug vor parole. In der Musik ähnlich wie in anderen Künsten ist das noetische Prinzip in seiner sinnlich-konkreten Einzel- 
haftigkeit untrennbar mit der individuellen Erfahrung des wahrnehmenden Subjekts verknüpft. Die künstlerische Wirklichkeitsaneignung hat subjektiven Charakter und findet seine Einsicht in die Wirklichkeit aufgrund des gesellschaftlich erreichbaren Intonationslexikons der Zeit, mit dem er schöpferisch arbeiten muß. Der noetische Prozeß interferiert somit zwischen individueller Erfahrung und allgemeiner Verständigung, zwischen Objektiven und Subjektiven.

Unterschiedlich ist auch das Verhältnis, welches das System der Sprache dem System erkannter objektiver Wirklichkeit gegenüber und das Verhältnis der Musik zur Wirklichkeit. Während das sprachliche System mit dem System erkannter Wirklichkeit kohärent ist, ist das System der Musik, gegründet auf spezifischer Verallgemeinerung der Wirklichkeit, unübersetzbar auf begriffliche Verallgemeinerung, mit dem System objektiver Realität unkohärent. Dies ist nachteilig für die Musik (sie vermißt »begriffliche Präzision« der Äußerung), doch anderseits arbeitet sie mit sinnlich konkreten Klangphänomenen und ist imstande komplizierte Prozesse menschlicher Psychik aufzufangen, die das Begriffsaparat nicht zu registrieren vermag. Sie weiß »das Unsagbare zu sagen«.

Der Grundunterschied besteht in der Widerspiegelung der Wirklichkeit in der Sprache und in der Musik. Während die Sprache sich der Wirklichkeit durch Benennung bemächtigt, verwendet die Musik direkt bestimmte Klangsegmente menschlicher Lebensempirie, sog. semantische Eintrittselemente. Diese manifestieren sich dann im System eines konkreten Einzelwerkes und reorganisieren sich zu Semantemen mit eigenen Bedeutungen und Inhalten. Es kommt so im Sinne der Theorie Asafjews zum Intonierungsprozeß, in dem bestimmte Intonationen die Wirksamkeit dynamischer semantischer Einheit annehmen, während andere sie verlieren und $\mathrm{zu}$ semantisch stabilisierten Einheiten - semantischen Eintrittselementen - werden.

Bei der Komparation der Sprache mit der Musik ist endlich jenes im Gedächtnis zu behalten, was überhaupt am wichtigsten ist, nämlich daß es sich um zwei ganz verschiedene Kommunikationssysteme handelt. Die Sprache ist das formellste und daher auch universalste Instrument der gesellschaflichen Kommunikation des Menschen. Die $\mathrm{Mu}-$ sik ist mehr als nur formelles Kommunikationsinstrument; sie schafft ähnlich wie andere Künste ein künstliches Milieu zwischen dem Menschen und der objektiven Realität, welches zur Aneignung und Vermenschlichung der Wirklichkeit und zur Umgestaltung des Menschen selbst dient.

Von allen diesen Vergleichen wird deutlich, daß man die Musik zwar als ein spezifisches semiotisches System betrachten kann, doch mit dem Zusatz, daß sie sich von der Sprache als künstlerisches System in mehreren wesentlichen Punkten absondert. Bei der Nutzung linguistischer Erkenntnisse müssen wir also höchst vorsichtig sein. 
Die bisherigen Ergebnisse namentlich in der allgemeinen Kunsttheorie schaffen Voraussetzungen dafür, damit wir eine Konstituierung des offenen musikalisch-semiotischen Systems versuchen. Im Hinblick darauf, daß die Musik ähnlich, wie andere Kunstarten eng mit der menschlichen Lebensempirie verknüpft ist, können wir von der Hypothese semantische Eintrittselemente ausgehen, die natürlich in jeder Kunstgattung spezifische Charakteristik innehaben. In der Musik kann man die semantischen Eintrittselemente schematisch in drei semantische Grundschichten einteilen:

1. natürliche und anthropologische,

2. der gesellschaftlichen Praxis,

3. der musikalischen Phylogenese.

Die natürliche und anthropologische Bedeutungsschicht wirkt z. B. bei der Klangorientierung in Zeit und Raum. In der Zeit orientiert uns der Klang durch seine Aufeinanderfolge, zeitliche Progressivität, im Raum durch seine Dynamik (Fernes und Nahes, Sich-Nähendes und Entfernendes), durch das Timbre informiert uns der Klang über seinen Urheber. Manche semantischen Eintrittselemente weisen in der Musik den Charakter anthropologischer Konstanten auf, vermittelt oft aufgrund der Synästhesie. Ein typisches Beispiel dafür bietet die Klanghöhe, synästhetisch mit der Spannung der Stimmbänder verknüpft. Diese Spannung wächst mit der Klangerhöhung, sinkt bei der Klangsenkung oder neutralisiert sich bei Beharrung auf bestimmter Hochebene. Mit der Höhe hängt die Lichtheit oder Dunkelheit des Klangs zusammen, die in der Komposition beim Registrieren zur Geltung kommen. Die Synästhesie hilft auch der Bedeutsamkeit der Intensität (Dynamik) des Klanges nach. Sehr starke Klänge wirken physiologisch schmerzhaft, stille Klänge hingegen tröstlich. Synästhetisch ist mit den Grundfunktionen des Lebens (Herzpuls, Atempulsierung) auch das Tempo verknüpft, der Rhythmus und das Metrum.

Aus der gesellschaftlichen Praxis tritt für die Musik in den Vordergrund die bedeutungsbildende Kraft der Produktionstätigkeit, die lebendige menschliche Sprache und des weiteren einige außermusikalische Kunstmanifestationen, besonders der Tanz. Was die Produktionstätigkeit betrifft, so hat bereits $\mathrm{K}$. Bücher in der berümhten $\mathrm{Ab}$ handlung "Arbeit und Rhythmus« (1896) auf die physiologisch und gesellschaftlich organisatorische Rolle des Rhythmus hiegewiesen, die vielleicht schon bei der Entstehung der Musik in primitiven Arbeitsgesangweisen Ausdruck gefunden hatte. Diese direkte Bindung lockerte sich allmählich (vor allem in der autonomen Musik), doch verschwand nicht völlig. Wir sind im Gegenteil in der Musik Zeugen eines stürmischen Einbruchs des "Maschinenrhythmus" in die autonome Musik, wo die ursprüngliche Bindung infolge des technischen 
Fortschrittes in eine andere Ebene transformiert ist. Eine nicht minder bedeutende, wenn auch kompliziert vermittelte Bindung besteht zwischen bestimmten Produktionsgeräten und der Musik. Die Waldhörner, Trompeten, Trommeln, Glocken usw. drangen in das Musikinstrumentarium aus der außermusikalischen Sphäre und tragen bis heute manchmal weniger deutliche, manchmal deutlichere Spuren ursprünglicher Bedeutungsfunktionen, mit denen der Komponist gezielt arbeitet.

Die Sprachmanifestation beeinflußte seit altersher auch die »musikalische Sprache«. Die Intonation der Rede als emotionaler »Subtext« des Gesprochenen mit einer fast unbegrenzten und doch fest legalisierten Menge von Veränderungen wurde den Komponisten nicht nur in der vokalen Musik, sondern auch in der instrumentalen zum Modell, ob sie sich schon bewußt (wie Leoš Janáček) oder vielmehr intuitiv nach diesen Modellen richteten.

Die uralte Verbindung der Musik mit dem Tanz, bzw. der Bewegung im allgemeinen ist $u$. a. auch ontisch gesetzt: beide Künste bewegen sich in Zeit und Raum. Daber können in die Musik leicht semantische Eintrittselemente aus den Bewegungskünsten strömen, doch aus dem Marsch (militärischen, Trauer- und Hochzeitsmarsch) udg. Die Kongruenz der Musik mit der Bewegungskunst, der Zeremonie usw. kann in der allgemeinsten Ebene an der gemeinsamen Antinomie der Kontinuität und Diskontinuität der Bewegung geltend gemacht werden. Der kontinuierliche Fluß schafft in der Musik ein Gefühl der Geschmeidigkeit (romantische melodische Linie) oder Monumentalität und Unendlichkeit (Wagner, Mahler). Die Diskontinuität kann Präzision und Abgemessenheit hervorrufen (Militärmarsch), doch auch Unruhe und Spannung. Eine genauere semantische Bestimmtheit hängt davon ab, in was für Kontexte in den einzelnen Fällen die semantischen Eintrittselemente gebracht werden, was eine allgemeingültige Maxime ist.

Zur Klärung der Bedeutungsschicht musikalischer Phylogenese ist es notwendig zu bemerken, daß die Grenzen zwischen semantischen Eintrittselementen und Semantemen sich stets verändert und daß die Semanteme, sobald sie genügend fixiert sind, die Rolle von semantischen Eintrittselementen übernehmen können. Diese aus der allgemeinen Theorie der Kunst übernommene Hypothese gilt auch für die Musik, Asafjew fang sie in dem Geschehen zwischen der Subintonations- und Intonationssphäre auf: bestimmte Intonationen verlieren den Charakter dynamisch-semantischer Einheit und funktionieren für derhin als Einheit mit einer nur potentiellen Bedeutung. Ein Elementarbeispiel dafür ist die allmähliche Verwischung einstiger Bedeutungen von bestimmten Intervallen, die somit ihre relative Selbständigkeit verloren haben und $\mathrm{zu}$ bloß partiellen Komponenten komplizierterer Intonationskomplexe geworden sind. Aus der Intonationssphäre sanken sie in die Subintonationssphäre hinab (ein klassischer Fall ist Tritonus - Diabolus in Musica). 


\begin{abstract}
Ähnlich wie in der Ebene der semantischen Eintrittselemente kann man das Spezifische der Musik auch in der Ebene der Semanteme untersuchen. Ein bestimmter Intervall, bzw. die Dinstanz zwischen einzelnen Bedeutungselementen innerhalb des Semantems und zwischen einem Semantem und der bisherigen Erfahrung des Hörers wird in der Musik ungefähr in vier Ebenen gelöst: des Genres, der
\end{abstract} Gattung, der tektonischen und stilmäßigen.

Das Wesen der Genreintonationen besteht darin, daß sie aus der funktionellen Anwendung der Musik in der Lebensempirie des Menschen vorwachsen. Die Gebundenheit an konkrete Lebenspraxis bewirkt, daß die Verbindung der Genreintonationen mit semantischen Eintrittselementen verhältnismäßig eng ist und deren gemeinsamer Intervall ein relativ statischer ist. Gattungsintonationen verdeutlichen semantisch die jeweiligen musikbildenden Möglichkeiten. Sie sind unmittelbar mit den Zeitformen des Musikverkehrs und dessen historisch-gesellschaftlicher Umwelt verknüpft. Die Richtung ihrer Intonationsprozesse geht von dem Gegebenen und semantisch »Natürlichen « zu dem vor, was möglich und semantisch erforderlich ist. In der Praxis wird solcher Prozeß einerseits z. B. durch Kombinationen von Klangbesetzung, Entstehung und Entfaltung der Virtuosität usw. und andererseits z. B. durch »Zum-Singen-Bringen « der Instrumente und hingegen Instrumentierung des vokalen Parts, durch gesichtete Destruktion der »natürlichen « Instrumentalität udg. Die Verbindung von Gattungsintonationen mit semantischen Eintrittselementen ist lockerer und deren gemeinsamer Intervall ist dynamischer. Tektonische Intonationen tragen Bedeutungen, die durch spezifische Musikgesetze des Aufbaus musikalischer Form vermittelt sind. Sie kommen besonders in autonomen Musikschaffen zur Geltung, wo sie eingehend individualisiert werden und der schaffenden Invention großen Raum bieten. Die Verbindung tektonischer Intonationen mit semantischen Eintrittselementen ist von allen Intonationsebene die lockerste. Deren gemeinsamer Intervall ist gleichfalls der dynamischeste. Die Stilintonationen zielen auf ganzheitliche Konzeption und einen Ausdruck der Situation des Menschen und dessen Rolle in seiner Epoche und stellen als solche eine dynamische Synthese aller Intonationsebenen dar. Für die Stilintonation wird bezeichnend, daß ihre Verbindung mit semantischen Eintrittselementen eine mittelbare ist, da es sich um einen hohen $\mathrm{Maß}$ an Verallgemeinerung handelt, doch zugleich beträchtlich komplex (als Synthese aller Intonationen). Die Beziehung zwischen Stilintonation und semantischen Eintrittselementen ist eine höchst dynamische (es geht um Verallgemeinerung), doch zugleich streng motiviert und in seiner Komplexheit relativ genau definiert.

Es leuchtet ein, daß in der unterbreiteten Konzeption der Begriffspaar Inhalt-Form ausbleibt. Gegenüber der traditionellen Auffassung werden Inhalt und Form als zwei Pole einer und derselben Erschein- 
ung, des Kunstwerks aufgefaßt, welches in dieser Zweipoligkeit den dynamischen Widerspruch seines Geschehens zutage fördert. Die eingeführten Begriffe »Eintrittselement der Bedeutung « und »Semantem« bilden eine Einheit inhaltlicher und formeller Elemente und strukturieren aufs neue die Ebene des Inhalts und der Form, oder genauer - der materiellen, semantischen und inhaltlichen Schicht des Kunstwerkes. Uns interessiert nun, worin das Spezifische des inhältlichen Geschehens im musikalischen Kunstwerk liegt.

So wie jeder Kunst so auch der Musik stehen einige Aspekte der Wirklichkeit näher, sie kann sie unmittelbar zum Ausdruck bringen, andere Aspekte liegen ihr ferner ab und sie muß sich ihnen mittelbar nähern. Die eigentliche Domäne der Musik ist das Prozessuale, Kontinuelle und die innere Bezugsverbindung der Wirklichkeit. $\mathrm{Zu}$ anderen Seiten muß sie durch jeweilige Vermittlung vordringen. In dialektischer Einheit des Unmittelbaren und Mittelbaren ist der Musik die objektive Realität in allen Grundkategorien der Wirklichkeitsrepräsentation zugänglich, was nach der bekannten Formel von Ch. Peirce die Zugänglichkeit in Indexvertretung (wo der inhaltliche Schwerpunkt der Musik als Ausdruckskunst liegt), in ikonischer (Klangmalerei, Bewegungscharakteristik, Nachahmung der Sprache) und symbolischer Vertretung (Zitationen, charakteristische Motive, Kryptogramme udg.), bzw. in einer ganzen Reihe fließen der Übergänge zwischen diesen Grundtypen bedeutet.

In der Frage des musikalischen Inhalts kommt es oft zu Verwechslungen mit dem Programm, ja zur Ablehnung der Inhaltlichkeit zugunsten der Programmhaftigkeit. Hier ist daran zu erinnern, daß das Programm der Musik von außen angefügt ist, während jedes musikalische Werk, dieses Namenswert, ob programmhaft oder nicht, Träger eines Inhalts ist. Davon überzeugt sich jeder Interpret oder Hörer, wenn sie hinter einer Notenaufzeichnung, bzw. Klängen und Geräuschen die Musik entdecken wollen, ein sinnvoles inhaltliches System. Ziel der psychischen Anstrengung ist nichts anderes als den Inhalt eines musikalischen Werkes zu entdecken.

Der musikalische Inhalt ist so wie die Inhaltsschicht jedes einzelnen Kunstwerkes ein offenes System, gegründet auf dialektischer Einheit musikalischer Phylo- und Ontogenese. Die phylogenetische Erfahrung ist jedoch im Bewußtssein des Hörers nie komplex gegenwärtig, in dem gesamten historisch-gesellschaftlichen Umfang. Sie ist konkret determiniert durch die Psychik der Zeit und die persönlichen Dispositionen des Interpreten oder Hörers. Daher kommt es zu immer neuen inhaltlichen Interpretationen. Die Tatsache, daß die Musik Inpretationskunst ist, tut den Prozeß inhaltlicher Aktualisierung und Individualisierung nur vervielfältigen.

Die Konfrontation einiger semiotischer Herangehen aus der allgemeinen Kunstheorie mit der musikologischen Problematik, die oben 
versucht wurde, stellt nur eine Andeutung von Möglichkeiten dar, die namentlich zur Klärung der Existenzgesetzmäßigkeit und das gesellschaftlichen Funktionierens eines musikalischen Kunstwerks verwendbar wären. Auf ersten Blick scheint es, daß das semiotische Herangehen vor allem für die Musiktheorie nutzbar sein kann und namentlich dann für die Klärung semantischen und inhaltlichen Geschehens des musikalischen Kunstwerkes. In der Tat sind die Möglichkeiten der Semiotik viel breiter und kommen in ihren Folgerungen auch in der Musikhistorie, Ethnomusikologie, Musiksoziologie udg. zur Geltung. Es ist neuerlich einmal daran zu erinnern, daß die Musiksemiotik erst im Stadium der Ausarbeitung sich befindet und daß sie noch nicht $\mathrm{zu}$ einer ganzheitlich selbständigen Disziplin der Musikwissenschaft geworden ist. Nichtsdestoweniger ist schon auf dieser Stufe deutlich, daß sie imstande sein wird, die weitere Entwicklung kognitiver musikalischer Reflexionen positiv zu beeinträchtigen.

\section{POVZETEK}

$\mathrm{S}$ pojmovanjem umetnosti kot sistema je poudarjena notranja in $\mathrm{zu}-$ nanja povezanost elementov kakor tudi dinamičnost umetnosti in njena stalna odprtost za širši sistem človeške eksistence. Poleg umetnika, dela in prejemnika so še drugi osnovni elementi sistema umetnosti, tako umetniška tradicija. Umetnosti je $\mathrm{v}$ korist, da približuje ustvarjalce in prejemnike na podlagi njihovega notranjega sorodstva, da poglablja odnos med njimi in medsebojno razumevanje ter na ta način ustvarja višje vsebinske sinteze.

Osnovno antinomijo glasbe lahko razumemo kot antinomijo intonacijskega leksikona dobe (langue) in čutno konkretnega muzikalnega izražanja (parole). Vendar se $\mathrm{v}$ glasbi ne da nobenega od pomenov ločiti od njene čutno konkretne zvočne forme. Komponist ne izbira le iz intonacijskega leksikona, ampak se zavzema za njegovo oddvojitev. Glasbena »govorica" ne izhaja iz splošnega »jezika« (langue), ampak deluje med individualnim izkustvom in splošnim sporazumevanjem. Sistem glasbe je za razliko od jezika $\mathrm{v}$ procesu spoznavanja inkoherenten $\mathrm{s}$ sistemom objektivnega sveta. Pogreša pojmovno preciznost, po drugi strani pa je sposoben zajeti zapletene procese človekove duševnosti, ki so pojmovnemu aparatu nedostopni. Osnovna razli'ka je $\mathrm{v}$ tem, da glasba $\mathrm{ni}$ le orodje, sredstvo za sporočanje, ampak da ustvarja med človekom in objektivno realnostjo tudi umetno okolje, ki služi za prisvajanje in humaniziranje resničnosti kakor tudi za preobražanje samega človeka.

Ce se zavedamo teh dejstev, se nam odpre možnost, da si ustvarimo okvirni glasbeno semiotični sistem vsaj na ravni semantičnih vstopnih elementov, ki vnašajo $\mathrm{v}$ delo segmente človekovega vsakdanjega doživljanja, in na ravni semantemov, novih posameznih pomenov, ki so lastni le delu.

V zgodovini estetike je postajalo vprašanje, kako pravilno zajeti glasbene vsebine, vse bolj komplicirano. To vprašanje pa je tudi dosti bolj zapleteno kot v katerikoli drugi umetnosti. Doslej ni našla zadovoljive rešitve niti marksistična glasbena estetika, čeprav je v svoji relativno kratki zgodovini prispevala marsikaj uspešnega. V zadnjem času je značilno zanimanje za spodbude s strani semiotike, s katero ima na gnoseološki ravni glede pojmovanja glasbe kot specifičnega 'komunikacijskega sredstva dosti skup- 
nega. V tej smeri se je že razvila intonacijska teorija B. Asafjeva in drugih raziskovalcev iz različnih dežel, med temi tudi čeških muzikologov, ki kritično navezujejo na strukturalistično šolo Mukařovskega. Njihovo središče je od 1971 praški interdisciplinarni team za preučevanje sistemov za izražanje in sporočanje umetnosti, $\mathrm{ki}$ ga vodi umetnostni zgodovinar S. Sabouk. Izhodiščna teza za koncept omenjenega teama je Marxova misel o umetnosti 'kot posebni obliki prisvajanja sveta po človeku. To funkcijo izpolnjuje umetnost s posebnim načinom sporočanja; ne obrača se na določeno posamezno funkcijo človeka kot npr. znanost, ampak na celoten kompleks njegovih bistvenih silnic (razum, čustvo, volja), ki jih reorganizira in spravlja $\mathrm{v}$ novo harmonijo.

Vsaka umetnina predstavlja podobo resničnosti, iz česar sledi, da poseduje znake. Vendar ni predmet umetnosti resničnost $\mathrm{v}$ njeni relativni avtonomiji, ampak počlovečena resničnost, $v$ katero se je odtisnil historično družbeni subjekt. Razlikovati pa moramo med predmetom, ki je lah'ko določen izrez resničnosti, in predmetom umetniškega odražanja, kateri predstavlja realnost človekove eksistence $\mathrm{v}$ njegovi specifični kvaliteti. Prva raven najde razlago tako $\mathrm{v}$ naravni plasti kakor tudi $\mathrm{v}$ družbeni in filogenetični. Druga je razporejena $\mathrm{v}$ intonaciji žanrov, zvrsti, $\mathrm{v}$ tektoniki in stilu. 